\title{
WEAK BEHAVIOUR OF FOURIER-NEUMANN SERIES
}

\author{
ÓSCAR CIAURRI \\ Departamento de Matemáticas y Computación, Universidad de La Rioja, Edificio J. L. Vives, \\ Calle Luis de Ulloas/n, 26004 Logroño, Spain \\ e-mail: oscar.ciaurri@dmc.unirioja.es
}

\section{MARIO PÉREZ}

Departamento de Matemáticas, Edificio de Matemáticas, Universidad de Zaragoza, 50009 Zaragoza, Spain e-mail:mperez@posta.unizar.es

\author{
and JUAN L. VARONA
}

Departamento de Matemáticas y Computación, Universidad de La Rioja, Edificio J. L. Vives,

Calle Luis de Ulloa s/n, 26004 Logroño, Spain

e-mail: jvarona@dmc.unirioja.es

URL: http://www.unirioja.es/dptos/dmc/jvarona/welcome.html

(Received 18 June, 2001; accepted 14 March, 2002)

\begin{abstract}
Let $J_{\mu}$ denote the Bessel function of order $\mu$. The functions $x^{-\alpha-1} J_{\alpha+2 n+1}(x), n=0,1,2, \ldots$, form an orthogonal system in the space $L^{2}((0, \infty)$, $x^{2 \alpha+1} d x$ ) when $\alpha>-1$. In this paper we prove that the Fourier series associated to this system is of restricted weak type for the endpoints of the interval of mean convergence, while it is not of weak type if $\alpha \geq 0$.
\end{abstract}

2000 Mathematics Subject Classification. 42C10, 44A05.

1. Introduction and results. Given a positive measure $\sigma$ on some space and an orthonormal system $\left\{\varphi_{n}\right\}_{n \geq 0}$ in $L^{2}(\sigma)$, the Fourier series associated to $\left\{\varphi_{n}\right\}_{n \geq 0}$ is the sequence of operators $S_{n}$ defined by

$$
S_{n} f=\sum_{k=0}^{n} c_{k}(f) \varphi_{k}, \quad f \in L^{2}(\sigma),
$$

where $c_{k}(f)=\int f \varphi_{k} d \sigma$. The elementary property that $\left\|S_{n} f-f\right\|_{L^{2}(\sigma)} \longrightarrow 0$ for every $f \in \overline{\operatorname{span}}\left\{\varphi_{n}\right\}_{n \geq 0}$ raises the same question with the $L^{2}(\sigma)$ norm replaced by the $L^{p}(\sigma)$ norm, $1 \leq p \leq \infty$. By the Banach-Steinhaus theorem, this is equivalent to the uniform boundedness

$$
\left\|S_{n} f\right\|_{L^{p}(\sigma)} \leq C\|f\|_{L^{p}(\sigma)}, \quad f \in L^{p}(\sigma), n \geq 0
$$

Needless to say, the most important case is the trigonometric system on the unit circle $\mathbb{T}$, for which the boundedness holds if $1<p<\infty$ [19]. For $p=\infty$ the answer is definitely negative, while for $p=1$ the boundedness fails but there is a weak substitute

Research supported by grants of the DGI and UR. 
in terms of the Lorentz space $L^{1, \infty}(\mathbb{T}, d \theta)$ :

$$
\left\|S_{n} f\right\|_{L^{1, \infty}(\mathbb{T}, d \theta)} \leq C\|f\|_{L^{1}(\mathbb{T}, d \theta)}, \quad f \in L^{1}(\mathbb{T}, d \theta), n \geq 0 .
$$

Here,

$$
\begin{gathered}
\|f\|_{L^{p, \infty}(\sigma)}=\sup _{y>0} y \lambda(y)^{1 / p}=\left\|t^{1 / p} f^{*}(t)\right\|_{L^{\infty}\left(\mathbb{R}^{+}, d t\right)}, \quad 1 \leq p<\infty, \\
\|f\|_{L^{, r}(\sigma)}=\left(\frac{r}{p} \int_{0}^{\infty}\left[t^{1 / p} f^{*}(t)\right]^{r} \frac{d t}{t}\right)^{1 / r}, \quad 1 \leq p<\infty, 1 \leq r<\infty,
\end{gathered}
$$

where $\lambda$ is the distribution function and $f^{*}$ the nonincreasing rearrangement of $f$. There is a Hölder's inequality $\|f\|_{p_{1}, p_{2}} \leq C\|f\|_{q_{1}, q_{2}}\|f\|_{r_{1}, r_{2}}, 1 / p_{i}=1 / q_{i}+1 / r_{i}$. Also, $\|f\|_{p, \infty} \leq C\|f\|_{p, p}=C\|f\|_{p} \leq C_{1}\|f\|_{p, 1}$. The reader is referred to [12] or [21] for further details on $L^{p, r}$ spaces.

After the trigonometric system, the convergence of Fourier series has been studied for a number of orthonormal systems, including Jacobi polynomials $[17,18,14,4,10]$, Hermite and Laguerre polynomials $[\mathbf{1 5}, \mathbf{1 6}]$, generalized Jacobi polynomials [1] and Bessel functions on $(0,1)[9]$.

In $[23,11]$ the authors characterized the $L^{p}$ convergence for the Fourier-Neumann series; i.e., the Fourier expansion associated to the functions

$$
\mathbf{j}_{n}^{\alpha}(x)=\sqrt{4 n+2 \alpha+2} J_{\alpha+2 n+1}(x) x^{-\alpha-1}, \quad n=0,1, \ldots
$$

which are orthonormal on $L^{2}\left((0, \infty), x^{2 \alpha+1} d x\right)\left[L^{2}\left(x^{2 \alpha+1}\right)\right.$, from now on]. See [24, $\S 13.41$ (7), p. 404] and [24, 13.42 (1), p. 405]. Here, $\alpha>-1$ and $J_{v}$ is the Bessel function of order $v$.

For each suitable function $f$, let $S_{n} f$ be the $n$-th partial sum of its Fourier series with respect to the system $\left\{\mathbf{j}_{n}^{\alpha}\right\}_{n=0}^{\infty}$; i.e.,

$$
S_{n}(f, x)=\int_{0}^{\infty} f(t) K_{n}(x, t) t^{2 \alpha+1} d t, \quad K_{n}(x, t)=\sum_{k=0}^{n} \mathbf{j}_{k}^{\alpha}(x) \mathbf{j}_{k}^{\alpha}(t) .
$$

In this paper, we study the weak and restricted weak behaviour of these series; i.e., the uniform boundedness

$$
\left\|S_{n} f\right\|_{L^{p, \infty}\left(x^{2 \alpha+1}\right)} \leq C\|f\|_{L^{p}\left(x^{2 \alpha+1}\right)}, \quad f \in L^{p}\left(x^{2 \alpha+1}\right), n \geq 0
$$

or

$$
\left\|S_{n} f\right\|_{L^{p, \infty}\left(x^{2 \alpha+1}\right)} \leq C\|f\|_{L^{p, 1}\left(x^{2 \alpha+1}\right)}, \quad f \in L^{p, 1}\left(x^{2 \alpha+1}\right), n \geq 0 .
$$

Let us focus on the weak boundedness. The a priori assumption that $\mathbf{j}_{n}^{\alpha} \in L^{q}\left(x^{2 \alpha+1}\right)$ $(n=0,1, \ldots, 1 / p+1 / q=1)$ should be made so as to guarantee the existence of the Fourier coefficients for any $f \in L^{p}\left(x^{2 \alpha+1}\right)$. Also, we must assume that $\mathbf{j}_{n}^{\alpha} \in L^{p, \infty}\left(x^{2 \alpha+1}\right)$ if we want $S_{n} f$ to be in $L^{p, \infty}\left(x^{2 \alpha+1}\right)$. By Lemmas 1 and 2 below, these assumptions hold if and only if $p_{1} \leq p<p_{2}$, where $p_{1}=4(\alpha+1) /(2 \alpha+3), p_{2}=4(\alpha+1) /(2 \alpha+1)$ if $\alpha \geq 0$, and $p_{1}=4 / 3, p_{2}=4$ if $-1<\alpha<0$. For the restricted weak boundedness, the same arguments lead to the a priori assumptions that $p_{1} \leq p \leq p_{2}$. Since the $L^{p}-L^{p}$ boundedness holds if and only if $p_{1}<p<p_{2}$, and it implies the $L^{p}-L^{p, \infty}$ and $L^{p, 1}-L^{p, \infty}$ 
boundedness, the real interest is in both endpoints. We obtain a negative answer for the weak type in the case $\alpha \geq 0$, and a positive answer for the restricted weak type.

THEOREM. Let $\alpha \geq 0, p_{1}=4(\alpha+1) /(2 \alpha+3), p_{2}=4(\alpha+1) /(2 \alpha+1)$. Then the partial sum operators $S_{n}, n=0,1, \ldots$, are not uniformly bounded as operators from $L^{p_{i}, 1}\left(x^{2 \alpha+1}\right)$ into $L^{p_{i}, \infty}\left(x^{2 \alpha+1}\right), i=1,2$. In the case $-1<\alpha<0$ the second statement holds with $p_{1}=4 / 3$ and $p_{2}=4$.

There is a close connection between $S_{n}$ and the Hankel transform $\mathcal{H}_{\alpha}$ given by

$$
\mathcal{H}_{\alpha} f(x)=\int_{0}^{\infty} \frac{J_{\alpha}(x y)}{(x y)^{\alpha}} f(y) y^{2 \alpha+1} d y .
$$

It turns out from [24, $\S 5.1$ (8), p. 134] and [7, proof of Lemma 4] that $S_{n} f=$ $\mathcal{H}_{\alpha}\left(\chi_{[0,1]} \mathcal{H}_{\alpha} f\right)-\mathcal{H}_{\alpha, n}\left(\chi_{[0,1]} \mathcal{H}_{\alpha, n} f\right) \equiv M_{\alpha} f-M_{\alpha, n} f$, where $\mathcal{H}_{\alpha, n}$ is given by (1) with $J_{\alpha+2 n+2}$ in place of $J_{\alpha}$. Now, Kenig and Tomas [13] proved that the multiplier $M_{\alpha}$ is not bounded from $L^{p}\left(x^{2 \alpha+1}\right)$ into $L^{p, \infty}\left(x^{2 \alpha+1}\right)$ when $p=4(\alpha+1) /(2 \alpha+3)$. Chanillo [5] proved that $M_{\alpha}$ is bounded from $L^{p, 1}\left(x^{2 \alpha+1}\right)$ into $L^{p, \infty}\left(x^{2 \alpha+1}\right)$ with $p=4(\alpha+1) /(2 \alpha+3)$. Some related uniform estimates were obtained by Carbery, Romera and Soria $[\mathbf{2 0}, 3]$ in the context of the disc multiplier.

Throughout this paper, unless otherwise stated, we use $C, C_{1}$ to denote positive constants independent of $n$ (and all other variables), that can assume different values in different occurrences. As usual, we write $f=O(g)$ in a given domain if $|f| \leq C g$. Finally, the standard notation $a_{+}=\max \{a, 0\}$ will be used.

2. Auxiliary results. Some appropriate estimates for Bessel functions will be needed. For instance,

$$
\begin{gathered}
J_{v}(x)=\frac{x^{v}}{2^{v} \Gamma(v+1)}+O\left(x^{\nu+2}\right), \quad x \rightarrow 0+, \\
J_{v}(x)=\sqrt{\frac{2}{\pi x}}\left[\cos \left(x-\frac{v \pi}{2}-\frac{\pi}{4}\right)+O\left(x^{-1}\right)\right], \quad x \rightarrow \infty
\end{gathered}
$$

where the $O$ terms depend on $v$. See [24, $\S 3.1$ (8), p. 40] and [24 $\S 7.21$ (1), p. 199]. Some bounds for $J_{v}$ and $J_{v}^{\prime}$ with constants independent of $v$ are also available. If $v>0,0<x \leq v / 2$ and $a \geq-v$, then there exists some constant $C_{a}$ depending only on $a$, such that

$$
\left|J_{v}(x)\right| x^{a} \leq C_{a} v^{a-1 / 2}\left(\frac{e}{4}\right)^{v}
$$

(see $\left[\mathbf{2 4}, \S 3.31\right.$, p. 49]). The formula $2 J_{v}^{\prime}=J_{v-1}-J_{v+1}$ proves the same bound for $J_{v}^{\prime}(x)$, as well as the analogs to (2) and (3):

$$
\begin{gathered}
J_{v}^{\prime}(x)=\frac{x^{\nu-1}}{2^{\nu} \Gamma(v)}+O\left(x^{\nu+1}\right), \quad x \rightarrow 0+, \\
J_{v}^{\prime}(x)=\sqrt{\frac{2}{\pi x}}\left[-\sin \left(x-\frac{v \pi}{2}-\frac{\pi}{4}\right)+O\left(x^{-1}\right)\right], \quad x \rightarrow \infty .
\end{gathered}
$$


It is easy to deduce from (4) and bounds done by Barceló and Córdoba (see [2, p. 661], $[8$, p. 24]) that

$$
\begin{array}{ccc}
\left|J_{v}(x)\right| \leq C x^{-1 / 4}\left(|x-v|+v^{1 / 3}\right)^{-1 / 4}, & x \in(0, \infty), \quad v>1 \\
\left|J_{v}^{\prime}(x)\right| \leq C x^{-3 / 4}\left(|x-v|+v^{1 / 3}\right)^{1 / 4}, & x \in(0, \infty), \quad v>1
\end{array}
$$

with some constant $C$ independent of $\nu$. As a consequence, the following estimate for the norm of $x^{b} J_{v}(x)$ and $x^{b} J_{v}^{\prime}(x)$ in $L^{p}\left(x^{2 \alpha+1}\right)$ and $L^{p, \infty}\left(x^{2 \alpha+1}\right)$ can be given.

Lemma 1. Let $1 \leq p<\infty, \alpha>-1, b \in R$ and $v>1$. Let $\lambda(4, v)=(\log v)^{1 / 4}$, $\lambda(p, v)=1$ if $p \neq 4$.

(a) $x^{b} J_{v}(x) \in L^{p}\left(x^{2 \alpha+1}\right)$ if and only if $p(b+v)+2 \alpha+2>0$ and $p\left(b-\frac{1}{2}\right)+2 \alpha+$ $2<0$. In this case, $\left\|x^{b} J_{v}(x)\right\|_{L^{p}\left(x^{2 \alpha+1}\right)} \leq C \lambda(p, v) v^{2 \frac{\alpha+1}{p}+b-\frac{1}{2}+\frac{2}{3}\left(\frac{1}{4}-\frac{1}{p}\right)+}$.

(b) $x^{b} J_{v}(x) \in L^{p, \infty}\left(x^{2 \alpha+1}\right)$ if and only if $p(b+v)+2 \alpha+2 \geq 0$ and $p\left(b-\frac{1}{2}\right)+2 \alpha+$ $2 \leq 0$. In this case, $\left\|x^{b} J_{v}(x)\right\|_{L^{p, \infty}\left(x^{2 \alpha+1}\right)} \leq C v^{2 \frac{\alpha+1}{p}+b-\frac{1}{2}+\frac{2}{3}\left(\frac{1}{4}-\frac{1}{p}\right)+}$.

(c) $x^{b} J_{v}^{\prime}(x) \in L^{p}\left(x^{2 \alpha+1}\right)$ if and only if $p(b+v-1)+2 \alpha+2>0$ and $p\left(b-\frac{1}{2}\right)+$ $2 \alpha+2<0$. In this case, $\left\|x^{b} J_{v}^{\prime}(x)\right\|_{L^{p}\left(x^{2 \alpha+1}\right)} \leq C v^{2 \frac{\alpha+1}{p}+b-\frac{1}{2}}$.

(d) $x^{b} J_{v}^{\prime}(x) \in L^{p, \infty}\left(x^{2 \alpha+1}\right)$ if and only if $p(b+v-1)+2 \alpha+2 \geq 0$ and $p\left(b-\frac{1}{2}\right)+$ $2 \alpha+2 \leq 0$. In this case, $\left\|x^{b} J_{\nu}^{\prime}(x)\right\|_{L^{p, \infty}\left(x^{2 \alpha+1}\right)} \leq C v^{2 \frac{\alpha+1}{p}+b-\frac{1}{2}}$.

Similar results can be found in $[\mathbf{2}, \mathbf{2 2}]$ and so we shall omit the proof. Details are given in [6, Chapter 2].) Let us just mention that the $L^{p}$ and $L^{p, \infty}$ conditions follow easily from (2), (3) and the analogs for $J_{v}^{\prime}(x)$, while the norm estimates are a consequence of (4), (5) and the analogs for $J_{v}^{\prime}(x)$.

Our next lemma is the main step in the proof of the uniform restricted weak type.

LEMMA 2. Let $v>1,1<p<\infty$, and $L_{v}(f, x)=J_{v}\left(x^{1 / 2}\right) H\left(t^{1 / 2} J_{v}^{\prime}\left(t^{1 / 2}\right) f(t), x\right)$, where $H$ denotes the Hilbert transform. There exists a constant $C$, independent of $v$, such that

(a) $\left\|L_{\nu} f\right\|_{L^{p}(d x)} \leq C\|f\|_{L^{p}(d x)}, f \in L^{p}(d x)$, if $p<4$,

(b) $\left\|L_{v} f\right\|_{L^{4, \infty}(d x)} \leq C\|f\|_{L^{4,1}(d x)}, f \in L^{4,1}(d x)$.

Proof. (a) It follows from (5) that

$$
\left\|L_{\nu} f\right\|_{L^{p}(d x)} \leq C\left\|H\left(t^{1 / 2} J_{v}^{\prime}\left(t^{1 / 2}\right) f(t), x\right)\right\|_{L^{p}\left(x^{-p / 8}\left(\left|x^{1 / 2}-\nu\right|+v^{1 / 3}\right)^{-p / 4}\right)} .
$$

Now, $x^{-p / 8}\left(\left|x^{1 / 2}-v\right|+v^{1 / 3}\right)^{-p / 4} \in A_{p}$ uniformly in $v$ if $p<4$. (See [7], [11] or [23].) Thus, $H$ is a bounded operator on $L^{p}\left(x^{-p / 8}\left(\left|x^{1 / 2}-v\right|+v^{1 / 3}\right)^{-p / 4}\right)$ and this, together with (6), proves (a).

(b) Let us write $L_{v}(f, x)=L_{v, 1}(f, x)+L_{v, 2}(f, x)$, where

$$
\begin{aligned}
& L_{v, 1}(f, x)=J_{v}\left(x^{1 / 2}\right)\left(\left|x^{1 / 2}-v\right|+v^{1 / 3}\right)^{1 / 4} H\left(\frac{t^{1 / 2} J_{v}^{\prime}\left(t^{1 / 2}\right) f(t)}{\left(\left|t^{1 / 2}-v\right|+v^{1 / 3}\right)^{1 / 4}}, x\right), \\
& L_{v, 2}(f, x)=J_{v}\left(x^{\frac{1}{2}}\right) H\left(\frac{t^{\frac{1}{2}} J_{v}^{\prime}\left(t^{\frac{1}{2}}\right) f(t)\left[\left(\left|t^{\frac{1}{2}}-v\right|+v^{\frac{1}{3}}\right)^{\frac{1}{4}}-\left(\left|x^{\frac{1}{2}}-v\right|+v^{\frac{1}{3}}\right)^{\frac{1}{4}}\right]}{\left(\left|t^{\frac{1}{2}}-v\right|+v^{\frac{1}{3}}\right)^{\frac{1}{4}}}, x\right) .
\end{aligned}
$$

The term $L_{v, 1}(f, x)$ is easy to handle: we have

$$
\left|J_{v}\left(x^{1 / 2}\right)\right|\left(\left|x^{1 / 2}-v\right|+v^{1 / 3}\right)^{1 / 4} \leq C x^{-1 / 8}
$$


and $x^{-1 / 2} \in A_{4}(0, \infty)$, so that

$$
\begin{aligned}
\left\|L_{v, 1} f\right\|_{L^{4, \infty}(d x)} & \leq C\left\|H\left(\frac{t^{1 / 2} J_{v}^{\prime}\left(t^{1 / 2}\right) f(t)}{\left(\left|t^{1 / 2}-v\right|+v^{1 / 3}\right)^{1 / 4}}, x\right)\right\|_{L^{4}\left(x^{-1 / 2}\right)} \\
& \leq C\left\|\frac{x^{1 / 2} J_{v}^{\prime}\left(x^{1 / 2}\right) f(x)}{\left(\left|x^{1 / 2}-v\right|+v^{1 / 3}\right)^{1 / 4}}\right\|_{L^{4}\left(x^{-1 / 2}\right)} \leq C\|f\|_{L^{4,1}(d x)} .
\end{aligned}
$$

Let us consider now $L_{v, 2} f$. The elementary inequality $\left|a^{1 / 4}-b^{1 / 4}\right| \leq a^{-3 / 4}|a-b|$, which holds for every $a, b>0$, gives

$$
\left|\left(\left|t^{\frac{1}{2}}-v\right|+v^{\frac{1}{3}}\right)^{\frac{1}{4}}-\left(\left|x^{\frac{1}{2}}-v\right|+v^{\frac{1}{3}}\right)^{\frac{1}{4}}\right| \leq t^{-1 / 2}\left(\left|t^{\frac{1}{2}}-v\right|+v^{\frac{1}{3}}\right)^{-\frac{3}{4}}|t-x| .
$$

Thus,

$$
\begin{aligned}
\left|L_{v, 2}(f, x)\right| & \leq\left|J_{v}\left(x^{1 / 2}\right)\right| \int_{0}^{\infty}\left|J_{v}^{\prime}\left(t^{1 / 2}\right)\right|\left(\left|t^{\frac{1}{2}}-v\right|+v^{\frac{1}{3}}\right)^{-1}|f(t)| d t \\
& \leq\left|J_{v}\left(x^{1 / 2}\right)\right|\left\|J_{v}^{\prime}\left(t^{1 / 2}\right)\left(\left|t^{\frac{1}{2}}-v\right|+v^{\frac{1}{3}}\right)^{-1}\right\|_{L^{4 / 3, \infty}(d t)}\|f\|_{L^{4,1}(d t)} .
\end{aligned}
$$

Finally, Lemma 1(b) and a small change in Lemma 1(d) give $\left\|J_{v}\left(x^{\frac{1}{2}}\right)\right\|_{L^{4, \infty}(d x)} \leq C$ and $\left\|J_{v}^{\prime}\left(x^{\frac{1}{2}}\right)\left(\left|x^{\frac{1}{2}}-v\right|+v^{\frac{1}{3}}\right)^{-1}\right\|_{L^{4 / 3, \infty(d x)}} \leq C$.

3. Weak boundedness. Using the fact that, for $\alpha>-1$, we have

$$
\begin{aligned}
\sum_{k=0}^{n} 2(\alpha+2 k+1) J_{\alpha+2 k+1}(x) J_{\alpha+2 k+1}(t)= & \frac{x t}{x^{2}-t^{2}}\left[x J_{\alpha+1}(x) J_{\alpha}(t)-t J_{\alpha}(x) J_{\alpha+1}(t)\right. \\
& +x J_{\alpha+2 n+2}^{\prime}(x) J_{\alpha+2 n+2}(t) \\
& \left.-t J_{\alpha+2 n+2}(x) J_{\alpha+2 n+2}^{\prime}(t)\right]
\end{aligned}
$$

(see $[\mathbf{1 1}, \mathbf{2 3}])$, we deduce that

$$
S_{n} f=W_{1} f-W_{2} f+W_{3, n} f-W_{4, n} f
$$

where

$$
\begin{aligned}
W_{1}(f, x) & =\frac{1}{2} x^{-\alpha+1} J_{\alpha+1}(x) H\left(t^{\alpha / 2} J_{\alpha}\left(t^{1 / 2}\right) f\left(t^{1 / 2}\right), x^{2}\right), \\
W_{2}(f, x) & =\frac{1}{2} x^{-\alpha} J_{\alpha}(x) H\left(t^{\alpha / 2+1 / 2} J_{\alpha+1}\left(t^{1 / 2}\right) f\left(t^{1 / 2}\right), x^{2}\right), \\
W_{3, n}(f, x) & =\frac{1}{2} x^{-\alpha+1} J_{v}^{\prime}(x) H\left(t^{\alpha / 2} J_{v}\left(t^{1 / 2}\right) f\left(t^{1 / 2}\right), x^{2}\right), \\
W_{4, n}(f, x) & =\frac{1}{2} x^{-\alpha} J_{v}(x) H\left(t^{\alpha / 2+1 / 2} J_{v}^{\prime}\left(t^{1 / 2}\right) f\left(t^{1 / 2}\right), x^{2}\right),
\end{aligned}
$$

and $v=\alpha+2 n+2$. Here, $H$ denotes the Hilbert transform on $(0, \infty)$. The following formulae were proved in [11, Theorem 1]:

$$
\begin{gathered}
\left\|W_{1} f\right\|_{L^{p}\left(x^{2 \alpha+1}\right)} \leq C\|f\|_{L^{p}\left(x^{2 \alpha+1}\right)}, \quad \frac{2 \alpha-1}{4(\alpha+1)}<\frac{1}{p}<\frac{2 \alpha+3}{4(\alpha+1)} \\
\left\|W_{2} f\right\|_{L^{p}\left(x^{2 \alpha+1}\right)} \leq C\|f\|_{L^{p}\left(x^{2 \alpha+1}\right)}, \quad \frac{2 \alpha+1}{4(\alpha+1)}<\frac{1}{p}<\frac{2 \alpha+5}{4(\alpha+1)}
\end{gathered}
$$




$$
\begin{aligned}
& \left\|W_{3, n} f\right\|_{L^{p}\left(x^{2 \alpha+1}\right)} \leq C\|f\|_{L^{p}\left(x^{2 \alpha+1}\right)}, \quad \frac{2 \alpha-\frac{1}{2}}{4(\alpha+1)}<\frac{1}{p}<\min \left\{\frac{2 \alpha+3}{4(\alpha+1)}, \frac{3}{4}\right\} ; \\
& \left\|W_{4, n} f\right\|_{L^{p}\left(x^{2 \alpha+1}\right)} \leq C\|f\|_{L^{p}\left(x^{2 \alpha+1}\right)}, \quad \max \left\{\frac{2 \alpha+1}{4(\alpha+1)}, \frac{1}{4}\right\}<\frac{1}{p}<\frac{2 \alpha+\frac{9}{2}}{4(\alpha+1)} .
\end{aligned}
$$

Now, let $\alpha \geq 0$. As mentioned in the introduction, the $S_{n}$ are not bounded operators from $L^{p}\left(x^{2 \alpha+1}\right)$ into $L^{p, \infty}\left(x^{2 \alpha+1}\right)$ if $p=4(\alpha+1) /(2 \alpha+1)$, so we only need to prove here that the uniform weak boundedness fails for $p=4(\alpha+1) /(2 \alpha+3)$.

It follows from (8) and (10) that $W_{2}$ and $W_{4, n}$ are uniformly bounded from $L^{p}\left(x^{2 \alpha+1}\right)$ into itself when $p=4(\alpha+1) /(2 \alpha+3)$. Thus, it will be enough to find a sequence of functions $\left\{f_{n}\right\}$ such that the inequality

$$
\left\|W_{1} f_{n}+W_{3, n} f_{n}\right\|_{L^{p, \infty}\left(x^{2 \alpha+1}\right)} \leq C\left\|f_{n}\right\|_{L^{p}\left(x^{2 \alpha+1}\right)}
$$

fails for every constant $C$. Let $f_{n}(t)=\operatorname{sgn}\left(J_{\alpha}(t)\right) t^{-\frac{2 \alpha+3}{2}} \chi_{[1, n]}(t)$. Then,

$$
\left\|f_{n}\right\|_{L^{p}\left(x^{2 \alpha+1}\right)}=C(\log n)^{\frac{1}{p}}, \quad p=4(\alpha+1) /(2 \alpha+3) .
$$

Now, for $v=\alpha+2 n+2$ and $x>2 v$ we have

$$
\left|W_{3, n}\left(f_{n}, x\right)\right| \leq C x^{-\alpha-1}\left|J_{v}^{\prime}(x)\right| \int_{1}^{n^{2}} t^{-\frac{3}{4}}\left|J_{\nu}\left(t^{\frac{1}{2}}\right)\right| d t \leq C x^{-\alpha-\frac{3}{2}}\left(\frac{e}{4}\right)^{2 n},
$$

where the last step follows from (6) and (4). Thus,

$$
\left\|\chi_{(2 v, \infty)} W_{3, n} f_{n}\right\|_{L^{p, \infty}\left(x^{2 \alpha+1}\right)} \leq C\left(\frac{e}{4}\right)^{2 n}, \quad p=4(\alpha+1) /(2 \alpha+3) .
$$

On the other hand, for $x>2 v$ we have

$$
\left|W_{1}\left(f_{n}, x\right)\right| \geq C x^{-\alpha-1}\left|J_{\alpha+1}(x)\right| \int_{1}^{n^{2}} t^{-\frac{3}{4}}\left|J_{\alpha}\left(t^{\frac{1}{2}}\right)\right| d t \geq C(\log n) x^{-\alpha-1}\left|J_{\alpha+1}(x)\right|,
$$

the last step following from (3). Therefore,

$$
\left\|\chi_{(2 v, \infty)}(x) W_{1}\left(f_{n}, x\right)\right\|_{L^{p, \infty}\left(x^{2 \alpha+1}\right)} \geq C \log n .
$$

Putting (12) and (13) together, we get

$$
\left\|W_{1} f_{n}+W_{3, n} f_{n}\right\|_{L^{p, \infty}\left(x^{2 \alpha+1}\right)} \geq C \log n, \quad p=4(\alpha+1) /(2 \alpha+3)
$$

and (11) indeed fails.

4. Restricted weak boundedness. By duality, we only need to prove that the self adjoint operators $S_{n}$ are uniformly of restricted weak type in two cases: $\alpha \geq 0, p=\frac{4(\alpha+1)}{2 \alpha+1}$, and $-1<\alpha<0, p=4$.

Case $\alpha \geq 0$ and $p=\frac{4(\alpha+1)}{2 \alpha+1}$. From (7) and (9), we conclude that $W_{1}$ and $W_{3, n}$ are uniformly bounded from $L^{p}\left(x^{2 \alpha+1}\right)$ into itself. Therefore, it is enough to prove that $W_{2}$ and $W_{4, n}$ are uniformly bounded from $L^{p, 1}\left(x^{2 \alpha+1}\right)$ into $L^{p, \infty}\left(x^{2 \alpha+1}\right)$; i.e.,

$$
\left\|W_{4, n} f\right\|_{L^{p, \infty}\left(x^{2 \alpha+1}\right)} \leq C\|f\|_{L^{p, 1}\left(x^{2 \alpha+1}\right)}, \quad f \in L^{p, 1}\left(x^{2 \alpha+1}\right)
$$


and a similar inequality for $W_{2}$. We shall consider only $W_{4, n}$, since the boundedness of $W_{2}$ is completely analogous. Let $f \in L^{p, 1}\left(x^{2 \alpha+1}\right)$ and, for each $k \in \mathbb{Z}$,

$$
I_{k}=\left[2^{k}, 2^{k+1}\right), \quad f_{1}^{k}=f \chi_{\left(0,2^{k-1}\right) \cup\left[2^{k+2}, \infty\right)}, \quad f_{2}^{k}=f \chi_{\left[2^{k-1}, 2^{k+2}\right)} .
$$

Thus, $f=f_{1}^{k}+f_{2}^{k}$ for each $k \in \mathbb{Z}$ and

$$
\left|W_{4, n}(f, x)\right| \leq \sum_{k \in \mathbb{Z}}\left|W_{4, n}\left(f_{1}^{k}, x\right)\right| \chi_{I_{k}}(x)+\sum_{k \in \mathbb{Z}}\left|W_{4, n}\left(f_{2}^{k}, x\right)\right| \chi_{I_{k}}(x) .
$$

Let $x \in I_{k}$. Then, it is easy to check that $\left|x^{2}-y^{2}\right| \geq \frac{3}{2} y^{2}$ if $y \in\left(0,2^{k-1}\right) \cup\left[2^{k+2,} \infty\right)$. Hence, after a change of variable we get

$$
\left|W_{4, n}\left(f_{1}^{k}, x\right)\right| \leq C x^{-\alpha}\left|J_{v}(x)\right| \int_{0}^{\infty} y^{\alpha}\left|J_{v}^{\prime}(y)\right||f(y)| d y
$$

and

$$
\sum_{k \in \mathbb{Z}}\left|W_{4, n}\left(f_{1}^{k}, x\right)\right| \chi_{I_{k}}(x) \leq C x^{-\alpha}\left|J_{\nu}(x)\right|\left\|x^{-\alpha-1} J_{\nu}^{\prime}(x)\right\|_{L^{q, \infty}\left(x^{2 \alpha+1}\right)}\|f\|_{L^{p, 1}\left(x^{2 \alpha+1}\right)},
$$

where $\frac{1}{p}+\frac{1}{q}=1$. Therefore, the first term in (14) is bounded:

$$
\left\|\sum_{k \in \mathbb{Z}}\left|W_{4, n}\left(f_{1}^{k}, x\right)\right| \chi_{I_{k}}(x)\right\|_{L^{p, \infty}\left(x^{2 \alpha+1}\right)} \leq C\|f\|_{L^{p, 1}\left(x^{2 \alpha+1}\right)}
$$

by Lemma 1(b) and (d). Let us consider now the second term. If $x \in I_{k}$,

$$
\left|W_{4, n}\left(f_{2}^{k}, x\right)\right| \leq C 2^{-\alpha k}\left|L_{\nu}\left(t^{\frac{\alpha}{2}} f_{k}^{2}\left(t^{1 / 2}\right), x^{2}\right)\right| .
$$

From Lemma 2 it follows that

$$
\begin{gathered}
\left\|W_{4, n}\left(f_{2}^{k}, x\right) \chi_{I_{k}}(x)\right\|_{L^{p, \infty}\left(x^{2 \alpha+1}\right)} \leq C 2^{-\alpha k+\frac{2 \alpha k}{p}}\left\|\chi_{I_{k}}(x) L_{v}\left(t^{\frac{\alpha}{2}} f_{k}^{2}\left(t^{1 / 2}\right), x^{2}\right)\right\|_{L^{p, \infty}(x d x)} \\
\leq C 2^{-\alpha k+\frac{2 \alpha k}{p}}\left\|x^{\frac{\alpha}{2}} f_{k}^{2}\left(x^{1 / 2}\right)\right\|_{L^{p, 1}(d x)} \leq C\left\|f \chi_{\left[2^{k-2}, 2^{k+2}\right)}\right\|_{L^{p, 1}\left(x^{2 \alpha+1}\right)}
\end{gathered}
$$

Then,

$$
\left\|\sum_{k \in \mathbb{Z}}\left|W_{4, n}\left(f_{2}^{k}, x\right)\right| \chi_{I_{k}}(x)\right\|_{L^{p, \infty}\left(x^{2 \alpha+1}\right)} \leq C\|f\|_{L^{p, 1}\left(x^{2 \alpha+1}\right)} .
$$

Case $-1<\alpha<0$ and $p=4$. Now, $W_{1}, W_{2}$, and $W_{3, n}$ are uniformly bounded from $L^{4}\left(x^{2 \alpha+1}\right)$ into itself (see (7), (8), (9)) and so we only need to prove that

$$
\left\|W_{4, n} f\right\|_{L^{4, \infty}\left(x^{2 \alpha+1}\right)} \leq C\|f\|_{L^{4,1}\left(x^{2 \alpha+1}\right)}, \quad f \in L^{4,1}\left(x^{2 \alpha+1}\right) .
$$

The above proof of (15) remains valid, while only minor changes are necessary for the first term in (14): it is not difficult to check that

$$
\frac{y^{2+\frac{\alpha}{2}} x^{-\frac{\alpha}{2}}}{\left|x^{2}-y^{2}\right|} \leq \frac{4}{3}
$$


if $x \in I_{k}$ and $y \in\left(0,2^{k-1}\right) \cup\left[2^{k+2}, \infty\right)$. Then, it follows that

$$
\left|W_{4, n}\left(f_{1}^{k}, x\right)\right| \leq C x^{-\frac{\alpha}{2}}\left|J_{v}(x)\right| \int_{0}^{\infty} y^{\frac{\alpha}{2}}\left|J_{v}^{\prime}(y)\right||f(y)| d y .
$$

\section{REFERENCES}

1. V. M. Badkov, Convergence in the mean and almost everywhere of Fourier series in polynomials orthogonal on an interval, Math. USSR Sb. 24 (1974), 223-256.

2. J. A. Barceló and A. Córdoba, Band-limited functions: $L^{p}$-convergence, Trans. Amer. Math. Soc. 313 (1989), 655-669.

3. A. Carbery, E. Romera and F. Soria, Radial weights and mixed norm inequalities for the disc multiplier, J. Funct. Anal. 109 (1992), 52-75.

4. S. Chanillo, On the weak behaviour of partial sums of Legendre series, Trans. Amer. Math. Soc. 268 (1981), 367-376. $18-24$.

5. S. Chanillo, The multiplier for the ball and radial functions, J. Funct. Anal. 55 (1984),

6. Ó. Ciaurri, Aproximación de funciones cuya transformada de Hankel está soportada en [0, 1], Ph. D. Thesis, University of La Rioja, Logroño (Spain), 2000.

7. Ó. Ciaurri, J. J. Guadalupe, M. Pérez and J. L. Varona, Mean and almost everywhere convergence of Fourier-Neumann series, J. Math. Anal. Appl. 236 (1999), 125-147.

8. A. Córdoba, The disc multiplier, Duke Math. J. 58 (1989), 21-29.

9. J. J. Guadalupe, M. Pérez, F. J. Ruiz and J. L. Varona, Mean and weak convergence of Fourier-Bessel series, J. Math. Anal. Appl. 173 (1993), 370-389.

10. J. J. Guadalupe, M. Pérez and J. L. Varona, Weak behaviour of Fourier-Jacobi series, J. Approx. Theory 61 (1990), 222-238.

11. J. J. Guadalupe, M. Pérez and J. L. Varona, Commutators and analytic dependence of Fourier-Bessel series on $(0, \infty)$, Canad. Math. Bull. 42 (1999), 198-208.

12. G. H. Hunt, On $L(p, q)$ spaces, Enseign. Math. 12 (1966), 249-276.

13. C. E. Kenig and P. A. Tomas, The weak behaviour of spherical means, Proc. Amer. Math. Soc. 78 (1980), 48-50.

14. B. Muckenhoupt, Mean convergence of Jacobi series, Proc. Amer. Math. Soc. 23 (1969), $306-310$

15. B. Muckenhoupt, Mean convergence of Hermite and Laguerre series. I, Trans. Amer. Math. Soc. 147 (1970), 419-431.

16. B. Muckenhoupt, Mean convergence of Hermite and Laguerre series. II, Trans. Amer. Math. Soc. 147 (1970), 433-460.

17. H. Pollard, The mean convergence of orthogonal series. II, Trans. Amer. Math. Soc. 63 (1948), 355-367.

18. H. Pollard, The mean convergence of orthogonal series. III, Duke Math. J. 16 (1949), $189-191$.

19. M. Riesz, Sur les fonctions conjuguées, Math. Z. 27 (1927), 218-244.

20. E. Romera and F. Soria, Endpoint estimates for the maximal operator associated to spherical partial sums on radial functions, Proc. Amer. Math. Soc. 111 (1991), 1015-1022.

21. E. M. Stein and G. Weiss, Introduction to Fourier analysis on Euclidean spaces (Princeton University Press, NJ, 1975).

22. K. Stempak, A weighted uniform $L^{p}$-estimate of Bessel functions: A note on a paper of Guo, Proc. Amer. Math. Soc. 128 (2000), 2943-2945.

23. J. L. Varona, Fourier series of functions whose Hankel transform is supported on [0, 1], Constr. Approx. 10 (1994), 65-75.

24. G. N. Watson, A treatise on the theory of Bessel functions (2nd edition) (Cambridge University Press, 1944). 